\title{
Oscillation Properties for Systems of Higher-Order Partial Differential Equations with Distributed Deviating Arguments
}

\author{
Youjun Liu, ${ }^{1,2}$ Jianwen Zhang, ${ }^{1}$ and Jurang Yan $^{3}$ \\ ${ }^{1}$ Institute of Applied Mechanics and Biomedical Engineering, Taiyuan University of Technology, Taiyuan, Shanxi 030024, China \\ ${ }^{2}$ College of Mathematics and Computer Sciences, Shanxi Datong University, Datong, Shanxi 037009, China \\ ${ }^{3}$ School of Mathematical Sciences, Shanxi University, Taiyuan, Shanxi 030006, China
}

Correspondence should be addressed to Jianwen Zhang; zjw9791@126.com

Received 13 June 2014; Accepted 15 December 2014

Academic Editor: Cengiz Çinar

Copyright (C) 2015 Youjun Liu et al. This is an open access article distributed under the Creative Commons Attribution License, which permits unrestricted use, distribution, and reproduction in any medium, provided the original work is properly cited.

New sufficient conditions are obtained for oscillation for the solutions of systems of a class of higher-order quasilinear partial functional differential equations with distributed deviating arguments. The obtained results are illustrated by example.

\section{Introduction and Preliminary}

In this paper, we consider systems of higher-order quasilinear partial functional differential equations with distributed deviating arguments:

$$
\begin{aligned}
\frac{\partial^{n}}{\partial t^{n}}\left[r(t) u_{i}(x, t)+\int_{\alpha}^{\beta} p(t, \tau) u_{i}(x, t-\tau) d \tau\right] \\
=a_{i}(t) \Delta u_{i}(x, t)+\sum_{j=1}^{m} \sum_{k=1}^{s} b_{i j k}(t) \Delta u_{j}\left(x, \rho_{k}(t)\right) \\
-q_{i}(x, t) u_{i}(x, t) \\
\quad-\sum_{j=1}^{m} \sum_{\gamma=1}^{l} \int_{\mathcal{C}_{\gamma}}^{d_{\gamma}} f_{i j \gamma}\left(x, t, u_{j}\left(x, t-\sigma_{\gamma}\right)\right) d \sigma_{\gamma}, \\
\quad(x, t) \in \Omega \times[0, \infty) \equiv G, \quad i=1,2, \ldots, m,
\end{aligned}
$$

where $n$ is even, $\Omega$ is a bounded domain in $R^{N}$ with a piecewise smooth boundary $\partial \Omega$, and

$$
\Delta u_{i}(x, t)=\sum_{r=1}^{N} \frac{\partial^{2} u_{i}(x, t)}{\partial x_{r}^{2}}, \quad i=1,2, \ldots, m .
$$

Throughout this paper, we assume that the following conditions hold:
(H1) $\beta>\alpha>0, d_{\gamma}>c_{\gamma}>0, r \in C^{n}([0, \infty),[0, \infty))$, $\lim _{t \rightarrow \infty} \int_{0}^{t}(1 / r(s)) d s=\infty ; p \in C^{n}([0, \infty) \times$ $[\alpha, \beta],[0, \infty)), \gamma \in I_{l}=\{1,2, \ldots, l\}$;

(H2) $q_{i} \in C(\bar{G},[0, \infty)), q_{i}(t)=\min _{x \in \bar{G}} q_{i}(x, t), q(t)=$ $\min _{1 \leq i \leq m} q_{i}(t), i \in I_{m}=\{1,2, \ldots, m\}$;

(H3) $f \in C(\bar{G} \times R, R), f_{i j \gamma}\left(t, x, u_{j}\right) / u_{j} \geq h_{i j \gamma}(x, t)$ if $u_{j} \neq 0$, where $h_{i j \gamma} \in C(\bar{G}, R), h_{i i \gamma}(x, t)>0$, $h_{i i \gamma}(t)=\min _{x \in \overline{\mathrm{G}}} h_{i i \gamma}(x, t), \bar{h}_{i j \gamma}(t)=\sup _{x \in \overline{\mathrm{G}}}\left|h_{i j \gamma}(x, t)\right|$, and $Q_{\gamma}(t)=\min _{1 \leq i \leq m}\left\{h_{i i \gamma}(t)-\sum_{j=1, j \neq i}^{m} \bar{h}_{i j \gamma}(t)\right\} \geq 0$, $i, j \in I_{m}, \gamma \in I_{l}$;

(H4) $a_{i} \in C([0, \infty),[0, \infty)), b_{i j k} \in C([0, \infty),(0, \infty))$, $B_{k}(t)=\min _{1 \leq i \leq m}\left\{b_{i i k}(t)-\sum_{j=1, j \neq i}^{m}\left|b_{i j k}(t)\right|\right\} \geq 0, i, j \in$ $I_{m}, k \in I_{s}=\{1,2, \ldots, s\}$;

(H5) $\rho_{k} \in C([0, \infty), R), \rho_{k}(t) \leq t, \rho_{k}$; is nondecreasing functions and $\lim _{t \rightarrow \infty} \rho_{k}(t)=\infty, k \in I_{s}$.

We consider two kinds of boundary conditions:

$$
\begin{gathered}
\frac{\partial u_{i}(x, t)}{\partial v}+g_{i}(x, t) u_{i}(x, t)=0, \\
(x, t) \in \partial \Omega \times[0, \infty), \quad i \in I_{m}
\end{gathered}
$$


where $v$ is the unit exterior normal vector to $\partial \Omega, g_{i}(x, t)$ is a nonnegative continuous function on $\partial \Omega \times[0, \infty)$, and

$$
u_{i}(x, t)=0, \quad(x, t) \in \partial \Omega \times[0, \infty), i \in I_{m} .
$$

During the past three decades, the investigation of oscillatory theory for partial functional differential equations has attracted attention of numerous researchers due to their significance in theory and applications. In 2000, Li [1] has investigated systems of second-order partial differential equations:

$$
\begin{aligned}
& \frac{\partial^{2}}{\partial t^{2}}\left[p(t) u_{i}(x, t)+\sum_{r=1}^{d} \lambda_{r}(t) u_{i}\left(x, t-\tau_{r}\right)\right] \\
& =a_{i}(t) \Delta u_{i}(x, t)+\sum_{j=1}^{m} \sum_{k=1}^{s} a_{i j k}(t) \Delta u_{j}\left(x, \rho_{k}(t)\right) \\
& \quad-q_{i}(x, t) u_{i}(x, t) \\
& \quad-\sum_{j=1}^{m} \sum_{h=1}^{l} q_{i j h}(x, t) u_{j}\left(x, \sigma_{h}(t)\right), \\
& (x, t) \in \Omega \times[0, \infty) \equiv G, \quad i=1,2, \ldots, m,
\end{aligned}
$$

and obtained sufficient conditions for the oscillation of solutions. In 2004, Lin [2] has studied higher-order nonlinear differential equation:

$$
\begin{aligned}
& \frac{\partial^{n}}{\partial t^{n}}\left[p(t) u_{i}(x, t)+\sum_{r=1}^{d} \lambda_{r}(t) u_{i}\left(x, t-\tau_{i}(t)\right)\right] \\
& =a_{i}(t) \Delta u_{i}(x, t)+\sum_{j=1}^{m} \sum_{k=1}^{s} a_{i j k}(t) \Delta u_{j}\left(x, \rho_{k}(t)\right) \\
& \quad-q_{i}(x, t) u_{i}(x, t) \\
& \quad-\sum_{j=1}^{m} \sum_{h=1}^{l} q_{i j h}(x, t) u_{j}\left(x, \sigma_{h}(t)\right), \\
& (x, t) \in \Omega \times[0, \infty) \equiv G, \quad i=1,2, \ldots, m,
\end{aligned}
$$

and obtained sufficient conditions for the oscillation of solutions. In 2004, Li et al. [3] have investigated a class of vector hyperbolic differential equations with deviating arguments:

$$
\begin{aligned}
\frac{\partial^{2}}{\partial t^{2}} U(x, t)= & a(t) \Delta U(x, t) \\
& +\sum_{k=1}^{m} b_{k}(t) \Delta U\left(x, \tau_{k}(t)\right)-p(x, t) U(x, t) \\
& -\sum_{h=1}^{l} \int_{a}^{b} q_{h}(x, t, \zeta) U\left(x, g_{h}(t, \zeta)\right) d \sigma(\zeta) \\
& +F(x, t), \quad(x, t) \in \Omega \times[0, \infty) \equiv G,
\end{aligned}
$$

and obtained sufficient conditions for $H$-oscillation of solutions. In 2009, Gui and Xu [4] have investigated even-order neutral type partial differential equations with distributed deviating arguments:

$$
\begin{gathered}
\frac{\partial}{\partial t}\left(p(t) \frac{\partial^{(m-1)}}{\partial t^{(m-1)}}\left[u(x, t)+c(t) u_{i}(x, t-\tau)\right]\right) \\
=a_{0}(t) \Delta u(x, t)+a_{1}(t) \Delta u(x, t-v) \\
-\int_{a}^{b} F(t, \xi, u(x, g(t, \xi))) d \mu(\xi), \\
(x, t) \in \Omega \times[0, \infty) \equiv G .
\end{gathered}
$$

For related work, we refer the reader to [5-10]. However, to the best of our knowledge, few investigitions have been reported on the oscillation theory of systems of partial functional differential equations with distributed deviating arguments. Thus, the research presents its significance.

Definition 1. The vector function $u(x, t)=\left\{u_{1}(x, t), u_{2}(x, t)\right.$, $\left.\ldots, u_{m}(x, t)\right\}^{\top}$ is said to be a solution of problem (1), (3) (or (1), (4)) if it satisfies (1) in $G=\Omega \times[0, \infty)$ and boundary condition (3) (or (4)).

Definition 2. A nontrivial component $u_{i}(x, t)$ of the vector function $u(x, t)=\left\{u_{1}(x, t), u_{2}(x, t), \ldots, u_{m}(x, t)\right\}^{\top}$ is said to oscillate in $\Omega \times\left[\mu_{0}, \infty\right)$ if, for each $\mu>\mu_{0}$, there is a point $\left(x_{0}, t_{0}\right) \in \Omega \times[\mu, \infty)$ such that $u_{i}\left(x_{0}, t_{0}\right)=0$.

Definition 3. The vector solution $u(x, t)=\left\{u_{1}(x, t), u_{2}(x, t)\right.$, $\left.\ldots, u_{m}(x, t)\right\}^{\top}$ of problem (1), (3) (or (1), (4)) is said to be oscillatory in $G=\Omega \times[0, \infty)$ if at least one of its nontrivial components is oscillatory in $G$. Otherwise, the vector solution $u(x, t)$ is said to be nonoscillatory.

\section{The Main Results}

Theorem 4. Assume that $r(t) \geq 1$ is a monotone decreasing function, and

$$
\int_{\alpha}^{\beta} p(t, \tau) d \tau<1 .
$$

If there exist some $\gamma_{0} \in I_{l}$ such that

$$
\begin{array}{r}
\int_{t_{1}}^{\infty}\left\{Q_{\gamma_{0}}(t) \int_{\mathcal{c}_{\gamma_{0}}}^{d_{\gamma_{0}}}\left[1-\int_{\alpha}^{\beta} p\left(t-\sigma_{\gamma_{0}}, \tau\right) d \tau\right] d \sigma_{\gamma_{0}}\right\} d t=\infty, \\
t_{0}>0,
\end{array}
$$

then every solution of boundary value system (1), (3) is oscillatory.

Proof. Suppose (1) and (3) have a nonoscillatory solution $\left\{u_{1}, u_{2}, \ldots, u_{m}\right\}$. We assume that $\left|u_{i}(x, t)\right|>0$ for $t \geq t_{0} \geq 0$, $i \in I_{m}$. Let $\delta_{i}=\operatorname{sgn} u_{i}(x, t), Z_{i}(x, t)=\delta_{i} u_{i}(x, t)$; then $Z_{i}(x, t)>0,(x, t) \in \Omega \times\left[t_{0}, \infty\right), i \in I_{m}$. From (H1) and 
Discrete Dynamics in Nature and Society

3

(H5), there exists a number $t_{1} \geq t_{0}$ such that $Z_{i}(x, t)>$ $0, Z_{i}(x, t-\tau)>0, Z_{i}\left(x, \rho_{k}(t)\right)>0, Z_{i}\left(x, t-\sigma_{\gamma}\right)>0$, in $\Omega \times\left[t_{0}, \infty\right), i \in I_{m}, k \in I_{s}, \gamma \in I_{l}$. Multiplying both sides of (1) by $\delta_{i}$ and integrating with respect to $x$ over the domain $\Omega$, we get

$$
\begin{aligned}
& \frac{d^{n}}{d t^{n}}\left[r(t) \int_{\Omega} \delta_{i} u_{i}(x, t) d x+\int_{\alpha}^{\beta} p(t, \tau) \int_{\Omega} \delta_{i} u_{i}(x, t-\tau) d x d \tau\right] \\
& =a_{i}(t) \int_{\Omega} \delta_{i} \Delta u_{i}(x, t) d x \\
& \quad+\sum_{j=1}^{m} \sum_{k=1}^{s} b_{i j k}(t) \int_{\Omega} \delta_{i} \Delta u_{j}\left(x, \rho_{k}(t)\right) d x \\
& \quad-\int_{\Omega} q_{i}(x, t) \delta_{i} u_{i}(x, t) d x \\
& \quad-\sum_{j=1}^{m} \sum_{\gamma=1}^{l} \int_{\Omega} \delta_{i} \int_{c_{\gamma}}^{d_{\gamma}} f_{i j \gamma}\left(x, t, u_{j}\left(x, t-\sigma_{\gamma}\right)\right) d \sigma_{\gamma} d x, \\
& \quad t \geq t_{1}, \quad i \in I_{m} .
\end{aligned}
$$

Therefore,

$$
\begin{aligned}
& \frac{d^{n}}{d t^{n}}\left[r(t) \int_{\Omega} Z_{i}(x, t) d x+\int_{\alpha}^{\beta} p(t, \tau) \int_{\Omega} Z_{i}(x, t-\tau) d x\right] d \tau \\
& \leq a_{i}(t) \int_{\Omega} \Delta Z_{i}(x, t) d x \\
& \quad+\sum_{j=1}^{m} \sum_{k=1}^{s} b_{i j k}(t) \int_{\Omega} \frac{\delta_{j}}{\delta_{i}} \Delta Z_{j}\left(x, \rho_{k}(t)\right) d x \\
& \quad-\int_{\Omega} q_{i}(x, t) Z_{i}(x, t) d x \\
& \quad-\sum_{j=1}^{m} \sum_{\gamma=1}^{l} \frac{\delta_{j}}{\delta_{i}} \int_{\mathcal{c}_{\gamma}}^{d_{\gamma}} \int_{\Omega} h_{i j \gamma}(x, t) Z_{j}\left(x, t-\sigma_{\gamma}\right) d x d \sigma_{\gamma}, \\
& t \geq t_{1}, \quad i \in I_{m} .
\end{aligned}
$$

From Green's formula and boundary condition (3), it follows that

$$
\begin{aligned}
\int_{\Omega} \Delta Z_{i}(x, t) d x & =\int_{\partial \Omega} \frac{\partial Z_{i}(x, t)}{\partial v} d S \\
& =-\int_{\partial \Omega} g_{i}(x, t) Z_{i}(x, t) d S \leq 0, \\
\int_{\Omega} \Delta Z_{i}\left(x, \rho_{k}(t)\right) d x & =\int_{\partial \Omega} \frac{\partial Z_{i}\left(x, \rho_{k}(t)\right)}{\partial v} d S \\
& =-\int_{\partial \Omega} g_{i}\left(x, \rho_{k}(t)\right) Z_{i}\left(x, \rho_{k}(t)\right) d S \\
& \leq 0,
\end{aligned}
$$

where $d S$ is the surface element on $\partial \Omega$. Combining (12)-(13), we find

$$
\begin{aligned}
& \frac{d^{n}}{d t^{n}}\left[r(t) \int_{\Omega} Z_{i}(x, t) d x+\int_{\alpha}^{\beta} p(t, \tau) \int_{\Omega} Z_{i}(x, t-\tau) d x\right] d \tau \\
& +\sum_{j=1}^{m} \sum_{k=1}^{s} b_{i j k}(t) \frac{\delta_{j}}{\delta_{i}} \int_{\partial \Omega} g_{i}\left(x, \rho_{k}(t)\right) Z_{i}\left(x, \rho_{k}(t)\right) d S \\
& +q_{i}(t) \int_{\Omega} Z_{i}(x, t) d x \\
& +\sum_{\gamma=1}^{l} h_{i i \gamma}(t) \int_{\mathcal{C}_{\gamma}}^{d_{\gamma}} \int_{\Omega} Z_{i}\left(x, t-\sigma_{\gamma}\right) d x d \sigma_{\gamma} \\
& \quad-\sum_{\gamma=1}^{l} \sum_{j=1, j \neq i}^{m} \bar{h}_{i j \gamma}(t) \int_{\mathcal{c}_{\gamma}}^{d_{\gamma}} \int_{\Omega} Z_{j}\left(x, t-\sigma_{\gamma}\right) d x d \sigma_{\gamma} \leq 0 .
\end{aligned}
$$

Therefore,

$$
\begin{aligned}
& \frac{d^{n}}{d t^{n}}\left[r(t) \int_{\Omega} Z_{i}(x, t) d x+\int_{\alpha}^{\beta} p(t, \tau) \int_{\Omega} Z_{i}(x, t-\tau) d \tau\right] d x \\
& +\sum_{k=1}^{s} b_{i i k}(t) \int_{\partial \Omega} g_{i}\left(x, \rho_{k}(t)\right) Z_{i}\left(x, \rho_{k}(t)\right) d S \\
& -\sum_{j=1, j \neq i}^{m} \sum_{k=1}^{s}\left|b_{i i k}(t)\right| \int_{\partial \Omega} g_{i}\left(x, \rho_{k}(t)\right) Z_{i}\left(x, \rho_{k}(t)\right) d S \\
& +q_{i}(t) \int_{\Omega} Z_{i}(x, t) d x \\
& +\sum_{\gamma=1}^{l} h_{i i \gamma}(t) \int_{\Omega} \int_{c_{\gamma}}^{d_{\gamma}} Z_{i}\left(x, t-\sigma_{\gamma}\right) d \sigma_{\gamma} d x \\
& -\sum_{\gamma=1}^{l} \sum_{j=1, j \neq i}^{m} \bar{h}_{i j \gamma}(t) \int_{\Omega} \int_{c_{\gamma}}^{d_{\gamma}} Z_{j}\left(x, t-\sigma_{\gamma}\right) d \sigma_{\gamma} d x \leq 0 .
\end{aligned}
$$

Set

$$
V_{i}=\int_{\Omega} Z_{i}(x, t) d x,
$$

$$
W_{i}=\int_{\partial \Omega} g_{i}\left(x, \rho_{k}(t)\right) Z_{i}\left(x, \rho_{k}(t)\right) d S,
$$

$$
t \geq t_{1}, \quad i \in I_{m} .
$$

Then (15) yields

$$
\begin{aligned}
& \frac{d^{n}}{d t^{n}}\left[r(t) V_{i}(t)+\int_{\alpha}^{\beta} p(t, \tau) V_{i}(t-\tau) d \tau\right] \\
& +\sum_{k=1}^{s}\left[b_{i i k}(t) W_{i}\left(\rho_{k}(t)\right)\right. \\
& \left.\quad-\sum_{j=1, j \neq i}^{m}\left|b_{i j k}(t)\right| W_{j}\left(\rho_{k}(t)\right)\right]+q_{i}(t) V_{i}(t)
\end{aligned}
$$




$$
\begin{aligned}
+\sum_{\gamma=1}^{l}\left[h_{i i \gamma}(t) \int_{c_{\gamma}}^{d_{\gamma}} V_{i}\left(t-\sigma_{\gamma}\right) d \sigma_{\gamma}\right. \\
\left.\quad-\sum_{j=1, j \neq i}^{m} \bar{h}_{i j \gamma}(t) \int_{c_{\gamma}}^{d_{\gamma}} V_{j}\left(t-\sigma_{\gamma}\right) d \sigma_{\gamma}\right] \leq 0 .
\end{aligned}
$$

Now, let $V(t)=\sum_{i=1}^{m} V_{i}(t), W(t)=\sum_{i=1}^{m} W_{i}(t), t \geq t_{1}$; from (17) we have

$$
\begin{aligned}
& \frac{d^{n}}{d t^{n}}\left[r(t) V(t)+\int_{\alpha}^{\beta} p(t, \tau) V(t-\tau) d \tau\right] \\
& +\sum_{k=1}^{s}\left\{\sum_{i=1}^{m}\left[b_{i i k}(t) W_{i}\left(\rho_{k}(t)\right)-\sum_{j=1, j \neq i}^{m}\left|b_{i j k}(t)\right| W_{j}\left(\rho_{k}(t)\right)\right]\right\} \\
& +q(t) V(t) \\
& +\sum_{\gamma=1}^{l}\left\{\sum _ { i = 1 } ^ { m } \left[h_{i i \gamma}(t) \int_{\mathcal{c}_{\gamma}}^{d_{\gamma}} V_{i}\left(t-\sigma_{\gamma}\right) d \sigma_{\gamma}\right.\right. \\
& \left.\left.-\sum_{j=1, j \neq i}^{m} \bar{h}_{i j \gamma}(t) \sum_{\gamma=1}^{l} \int_{\mathcal{c}_{\gamma}}^{d_{\gamma}} V_{j}\left(t-\sigma_{\gamma}\right) d \sigma_{\gamma}\right]\right\} \leq 0 .
\end{aligned}
$$

Note that

$$
\begin{aligned}
& \sum_{i=1}^{m}\left\{h_{i i \gamma}(t) \int_{c_{\gamma}}^{d_{\gamma}} V_{i}\left(t-\sigma_{\gamma}\right) d \sigma_{\gamma}\right. \\
& \left.-\sum_{j=1, j \neq i}^{m} \bar{h}_{i j \gamma}(t) \int_{c_{\gamma}}^{d_{\gamma}} V_{j}\left(t-\sigma_{\gamma}\right) d \sigma_{\gamma}\right\} \\
& =\left[h_{11 \gamma}(t) \int_{c_{\gamma}}^{d_{\gamma}} V_{1}\left(t-\sigma_{\gamma}\right) d \sigma_{\gamma}\right. \\
& \left.-\sum_{j=1, j \neq 1}^{m} \bar{h}_{1 j \gamma}(t) \int_{\mathcal{c}_{\gamma}}^{d_{\gamma}} V_{j}\left(t-\sigma_{\gamma}\right) d \sigma_{\gamma}\right] \\
& +\left[h_{22 \gamma}(t) \int_{c_{\gamma}}^{d_{\gamma}} V_{2}\left(t-\sigma_{\gamma}\right) d \sigma_{\gamma}\right. \\
& \left.-\sum_{j=1, j \neq 2}^{m} \bar{h}_{2 j \gamma}(t) \int_{c_{\gamma}}^{d_{\gamma}} V_{j}\left(t-\sigma_{\gamma}\right) d \sigma_{\gamma}\right] \\
& +\cdots+\left[h_{m m \gamma}(t) \int_{\mathcal{c}_{\gamma}}^{d_{\gamma}} V_{m}\left(t-\sigma_{\gamma}\right) d \sigma_{\gamma}\right. \\
& \left.-\sum_{j=1, j \neq m}^{m} \bar{h}_{m j \gamma}(t) \int_{\mathcal{c}_{\gamma}}^{d_{\gamma}} V_{j}\left(t-\sigma_{\gamma}\right) d \sigma_{\gamma}\right]
\end{aligned}
$$

$$
\begin{aligned}
= & {\left[h_{11 \gamma}(t)-\sum_{j=1, j \neq 1}^{m} \bar{h}_{j 1 \gamma}(t)\right] \int_{\mathcal{c}_{\gamma}}^{d_{\gamma}} V_{1}\left(t-\sigma_{\gamma}\right) d \sigma_{\gamma} } \\
& +\left[h_{22 \gamma}(t)-\sum_{j=1, j \neq 1}^{m} \bar{h}_{j 2 \gamma}(t)\right] \int_{\mathcal{c}_{\gamma}}^{d_{\gamma}} V_{2}\left(t-\sigma_{\gamma}\right) d \sigma_{\gamma} \\
& +\cdots+\left[h_{m m \gamma}(t)-\sum_{j=1, j \neq 1}^{m} \bar{h}_{j m \gamma}(t)\right] \int_{\mathcal{c}_{\gamma}}^{d_{\gamma}} V_{m}\left(t-\sigma_{\gamma}\right) d \sigma_{\gamma} \\
\geq & \min _{1 \leq i \leq m}\left\{h_{i i \gamma}(t)-\sum_{j=1, j \neq i}^{m} \bar{h}_{j i \gamma}(t)\right\} \sum_{i=1}^{m} \int_{\mathcal{c}_{\gamma}}^{d_{\gamma}} V_{i}\left(t-\sigma_{\gamma}\right) d \sigma_{\gamma} \\
= & Q_{\gamma}(t) \int_{\mathcal{C}_{\gamma}}^{d_{\gamma}} V\left(t-\sigma_{\gamma}\right) d \sigma_{\gamma} .
\end{aligned}
$$

Similarly, we have

$$
\begin{aligned}
& \sum_{k=1}^{s}\left\{\sum_{i=1}^{m}\left[b_{i i k}(t) W_{i}\left(\rho_{k}(t)\right)-\sum_{j=1, j \neq i}^{m}\left|b_{i j k}(t)\right| W_{j}\left(\rho_{k}(t)\right)\right]\right\} \\
& \quad \geq \min _{1 \leq i \leq m}\left\{b_{i i k}(t)-\sum_{j=1, j \neq i}^{m}\left|b_{j i k}(t)\right|\right\} \sum_{i=1}^{m} W_{i}\left(\rho_{k}(t)\right) \\
& =B_{k}(t) W\left(\rho_{k}(t)\right) .
\end{aligned}
$$

Thus, from (18) for $t \geq t_{1}$, we find

$$
\begin{aligned}
& {\left[r(t) V(t)+\int_{\alpha}^{\beta} p(t, \tau) V(t-\tau) \mathrm{d} \tau\right]^{(n)}} \\
& +\sum_{k=1}^{s} B_{k}(t) W\left(\rho_{k}(t)\right)+q(t) V(t) \\
& \quad+\sum_{\gamma=1}^{l} Q_{\gamma}(t) \int_{c_{\gamma}}^{d_{\gamma}} V\left(t-\sigma_{\gamma}\right) d \sigma_{\gamma} \leq 0, \quad t \geq t_{1} .
\end{aligned}
$$

It is clear that

$$
W\left(\rho_{k}(t)\right)=\sum_{i=1}^{m} W_{i}\left(\rho_{k}(t)\right) \geq 0, \quad t \geq t_{1}, k \in I_{s} .
$$

Therefore, it follows that

$$
\begin{aligned}
& {\left[r(t) V(t)+\int_{\alpha}^{\beta} p(t, \tau) V(t-\tau) d \tau\right]^{(n)}} \\
& \quad+q(t) V(t)+\sum_{\gamma=1}^{l} Q_{\gamma}(t) \int_{\mathcal{C}_{\gamma}}^{d_{\gamma}} V\left(t-\sigma_{\gamma}\right) d \sigma_{\gamma} \leq 0,
\end{aligned}
$$


Let

$$
Y(t)=r(t) V(t)+\int_{\alpha}^{\beta} p(t, \tau) V(t-\tau) d \tau .
$$

We have $Y(t)>0, Y^{(n)}(t)<0$, for $t \geq t_{1}$. Hence, $Y^{(n-1)}(t)$ is a monotone decreasing function in the interval $\left[t_{1}, \infty\right)$. We can claim that $Y^{(n-1)}(t)>0$, for $t \geq t_{1}$. In fact, if $Y^{(n-1)}(t)<0$, for $t \geq t_{1}$, then there exists a $T>t_{1}$, such that $r(T) Y^{(n-1)}(T)<0$. This implies that

$$
Y^{(k)}(t)<0, \quad k=0,1,2, \ldots, n-2,
$$

which contradicts the fact that $Y(t)>0$. Now, we claim that $Y^{\prime}(t) \geq 0, t \geq t_{1}$. Otherwise $Y(t)<0$. But from the wellknown lemma of Kiguradze [5] and the fact that $n$ is even, the inequalities imply that higher derivatives of that function are also negative. This contradicts the fact that $Y^{(n-1)}(T) \geq 0$. Thus we have proved that $Y(t)$ is the increasing function of $t$.

From (23), we find that there exists a $\gamma_{0} \in I_{l}$ such that

$$
Y^{(n)}(t)+Q_{\gamma_{0}}(t) \int_{\mathcal{c}_{\gamma_{0}}}^{d_{\gamma_{0}}} V\left(t-\sigma_{\gamma_{0}}\right) d \sigma_{\gamma_{0}} \leq 0, \quad t \geq t_{1} .
$$

Thus we obtain

$$
\begin{aligned}
Y^{(n)}(t)+Q_{\gamma_{0}}(t) & \\
\times \int_{\mathcal{c}_{\gamma_{0}}}^{d_{\gamma_{0}}}([ & {\left[\left(t-\sigma_{\gamma_{0}}\right)\right.} \\
& \left.\quad-\int_{\alpha}^{\beta} p\left(t-\sigma_{\gamma_{0}}, \tau\right) V\left(t-\sigma_{\gamma_{0}}-\tau\right) d \tau\right] \\
& \left.\times\left(r\left(t-\sigma_{\gamma_{0}}\right)\right)^{-1}\right) d \sigma_{\gamma_{0}}
\end{aligned}
$$

$\leq 0, \quad t \geq t_{1}$.

Since $Y(t) \geq r(t) V(t) \geq V(t), Y^{\prime}(t) \geq 0$, from (27), we have

$$
\begin{array}{r}
Y^{(n)}(t)+Q_{\gamma_{0}}(t) \\
\times \int_{\mathcal{C}_{\gamma_{0}}}^{d_{\gamma_{0}}}\left[1-\int_{\alpha}^{\beta} p\left(t-\sigma_{\gamma_{0}}, \tau\right) d \tau\right] \frac{Y\left(t-\sigma_{\gamma_{0}}\right)}{r\left(t-\sigma_{\gamma_{0}}\right)} d \sigma_{\gamma_{0}} \leq 0, \\
t \geq t_{1} .
\end{array}
$$

Integrating inequality (28) from $t_{1}$ to $t$,

$$
\begin{aligned}
Y^{(n-1)}(t)-Y^{(n-1)}\left(t_{1}\right) & \\
+\int_{t_{1}}^{t}\left\{Q_{\gamma_{0}}(s) \int_{\mathcal{c}_{\gamma_{0}}}^{d_{\gamma_{0}}}\right. & {\left[1-\int_{\alpha}^{\beta} p\left(s-\sigma_{\gamma_{0}}, \tau\right) d \tau\right] } \\
& \left.\times \frac{Y\left(t-\sigma_{\gamma_{0}}\right)}{r\left(t-\sigma_{\gamma_{0}}\right)} d \sigma_{\gamma_{0}}\right\} d s \leq 0, \quad t \geq t_{1} .
\end{aligned}
$$

Furthermore, we have

$$
\begin{aligned}
& \int_{t_{1}}^{t}\left\{Q_{\gamma_{0}}(s) \int_{\mathcal{c}_{\gamma_{0}}}^{d_{\gamma_{0}}}\left[1-\int_{\alpha}^{\beta} p\left(s-\sigma_{\gamma_{0}}, \tau\right) d \tau\right] d \sigma_{\gamma_{0}}\right\} d s \\
& \quad \leq \frac{r\left(t_{1}-d_{\gamma_{0}}\right)}{Y\left(t_{1}-d_{\gamma_{0}}\right)}\left(-Y^{(n-1)}(t)+Y^{(n-1)}\left(t_{1}\right)\right), \\
& \quad \leq \frac{r\left(t_{1}-d_{\gamma_{0}}\right) Y^{(n-1)}\left(t_{1}\right)}{Y\left(t_{1}-d_{\gamma_{0}}\right)}, \quad t \geq t_{1},
\end{aligned}
$$

which contradicts condition (10). This proof is complete.

Theorem 5. Assume that $r(t) \geq 1$ is a monotone decreasing function and (9) holds. And

$$
\int_{t_{0}}^{\infty}\left\{q(s)\left[1-\int_{\alpha}^{\beta} p(s, \tau) d \tau\right]\right\} d s=\infty, \quad t_{0}>0 .
$$

Then every solution of boundary value system (1), (3) is oscillatory.

Proof. As in the proof of Theorem 4, we obtain (23). Therefore,

$$
Y^{(n)}(t)+q(t) V(t) \leq 0, \quad t \geq t_{0} .
$$

The remaining part of the proof is similar to that of Theorem 4; therefore it is omitted. The proof is complete.

Corollary 6. If inequality (23) has no eventually positive solution, then every solution $u(x, t)$ of system (1), (3) is oscillatory in $G$.

Theorem 7. Suppose that there exists a positive constant $M$ such that $0<M \leq r(t)<1$, and

$$
\int_{\alpha}^{\beta} p(t, \tau) d \tau<M
$$

If there exist some $\gamma_{0} \in I_{l}$ such that

$$
\begin{aligned}
\int_{t_{1}}^{\infty} & \left\{Q_{\gamma_{0}}(t) \int_{\mathcal{c}_{\gamma_{0}}}^{d_{\gamma_{0}}}\left[1-M^{-1} \int_{\alpha}^{\beta} p\left(t-\sigma_{\gamma_{0}}, \tau\right) d \tau\right] d \sigma_{\gamma_{0}}\right\} d t \\
= & \infty, \quad t_{0}>0,
\end{aligned}
$$

then every solution of boundary value system (1), (3) is oscillatory. 
Proof. As in the proof of Theorem 4, we obtain (27). Using the fact that $r(t)<1$, from (27), we have

$$
\begin{aligned}
Y^{(n)}(t)+ & Q_{\gamma_{0}}(t) \\
\times \int_{c_{\gamma_{0}}}^{d_{\gamma_{0}}} & {\left[Y\left(t-\sigma_{\gamma_{0}}\right)\right.} \\
\quad & \left.\quad \int_{\alpha}^{\beta} p\left(t-\sigma_{\gamma_{0}}, \tau\right) V\left(t-\sigma_{\gamma_{0}}-\tau\right) d \tau\right] d \sigma_{\gamma_{0}}
\end{aligned}
$$

$\leq 0, \quad t \geq t_{1}$.

Since $Y(t) \geq r(t) V(t) \geq M V(t), Y^{\prime}(t) \geq 0$, from (35) we have

$$
\begin{aligned}
& Y^{(n)}(t)+Q_{\gamma_{0}}(t) \\
& \quad \times \int_{\mathcal{c}_{\gamma_{0}}}^{d_{\gamma_{0}}}\left[1-M^{-1} \int_{\alpha}^{\beta} p\left(t-\sigma_{\gamma_{0}}, \tau\right) d \tau\right] Y\left(t-\sigma_{\gamma_{0}}\right) d \sigma_{\gamma_{0}} \\
& \leq 0, \quad t \geq t_{1} .
\end{aligned}
$$

Integrating inequality (36) from $t_{1}$ to $t$,

$$
\begin{array}{r}
Y^{(n-1)}(t)-Y^{(n-1)}\left(t_{1}\right) \\
+\int_{t_{1}}^{t}\left\{Q_{\gamma_{0}}(s) \int_{\mathcal{c}_{\gamma_{0}}}^{d_{\gamma_{0}}}\left[1-M^{-1} \int_{\alpha}^{\beta} p\left(s-\sigma_{\gamma_{0}}, \tau\right) d \tau\right]\right. \\
\left.\times Y\left(t-\sigma_{\gamma_{0}}\right) d \sigma_{\gamma_{0}}\right\} d s \leq 0, \\
t \geq t_{1} .
\end{array}
$$

Furthermore, we have

$$
\begin{aligned}
& \int_{t_{1}}^{t}\left\{Q_{\gamma_{0}}(s) \int_{\mathcal{c}_{\gamma_{0}}}^{d_{\gamma_{0}}}\left[1-M^{-1} \int_{\alpha}^{\beta} p\left(s-\sigma_{\gamma_{0}}, \tau\right) d \tau\right] d \sigma_{\gamma_{0}}\right\} d s \\
& \leq \frac{\left(-Y^{(n-1)}(t)+Y^{(n-1)}\left(t_{1}\right)\right)}{Y\left(t_{1}-d_{\gamma_{0}}\right)}, \\
& \leq \frac{Y^{(n-1)}\left(t_{1}\right)}{Y\left(t_{1}-d_{\gamma_{0}}\right)}, \quad t \geq t_{1},
\end{aligned}
$$

which contradicts condition (10). This proof is complete.

Theorem 8. Suppose that there exists a positive constant $M$ such that $0<M \leq r(t)<1$, and (33) holds. If

$$
\int_{t_{0}}^{\infty}\left\{q(s)\left[1-M^{-1} \int_{\alpha}^{\beta} p(s, \tau) d \tau\right]\right\} d s=\infty, \quad t_{0}>0,
$$

then every solution of boundary value system (1), (3) is oscillatory.
Proof. As in the proof of Theorem 5, we obtain (32). The remaining part of the proof is similar to that of Theorem 7 and we omit it.

Consider the smallest eigenvalue $\lambda_{0}$ of the Dirichlet problem:

$$
\begin{gathered}
\Delta \omega(x)+\lambda \omega(x)=0, \quad x \in \Omega, \\
\omega(x)=0, \quad x \in \partial \Omega,
\end{gathered}
$$

where $\lambda$ is a positive constant and the corresponding eigenfunction $\phi(x)$ is positive in $\Omega$.

Theorem 9. Assume all conditions of Theorem 4 hold. Then every solution $u(x, t)$ of system (1), (4) is oscillatory in $G$.

Proof. Suppose (1) and (4) have a nonoscillatory solution $\left\{u_{1}, u_{2}, \ldots, u_{m}\right\}^{T}$. We assume that $\left|u_{i}(x, t)\right|>0$ for $t \geq t_{0} \geq$ $0, i \in I_{m}$. Let $\delta_{i}=\operatorname{sgn} u_{i}(x, t), Z_{i}(x, t)=\delta_{i} u_{i}(x, t)$; then $Z_{i}(x, t)>0,(x, t) \in \Omega \times\left[t_{0}, \infty\right), i \in I_{m}$. From $(H 1)$ and (H5), there exists a number $t_{1} \geq t_{0}$ such that $Z_{i}(x, t)>$ $0, Z_{i}(x, t-\tau)>0, Z_{i}\left(x, \rho_{k}(t)\right)>0, Z_{i}\left(x, t-\sigma_{\gamma}\right)>0$, in $\Omega \times\left[t_{0}, \infty\right), i \in I_{m}, k \in I_{s}, \gamma \in I_{l}$. Multiplying both sides of (1) by $\delta_{i} \phi(x)$, and integrating with respect to $x$ over the domain $\Omega$, we have

$$
\begin{aligned}
& \frac{d^{n}}{d t^{n}}\left[r(t) \int_{\Omega} \delta_{i} u_{i}(x, t) \phi(x) d x\right. \\
& \left.\quad+\int_{\alpha}^{\beta} p(t, \tau) \int_{\Omega} \delta_{i} u_{i}(x, t-\tau) \phi(x) d x d \tau\right] \\
& =a_{i}(t) \int_{\Omega} \delta_{i} \phi(x) \Delta u_{i}(x, t) d x \\
& \quad+\sum_{j=1}^{m} \sum_{k=1}^{s} b_{i j k}(t) \int_{\Omega} \delta_{i} \phi(x) \Delta u_{j}\left(x, \rho_{k}(t)\right) d x \\
& \quad-\int_{\Omega} q_{i}(x, t) \delta_{i} u_{i}(x, t) \phi(x) d x \\
& \quad-\sum_{\gamma=1}^{l} \int_{\Omega} \delta_{i} \phi(x) \int_{c_{\gamma}}^{d_{\gamma}} f_{i j \gamma}\left(x, t, u_{j}\left(x, t-\sigma_{\gamma}\right)\right) d \sigma_{\gamma} d x, \\
& \quad t \geq t_{1}, \quad i \in I_{m} .
\end{aligned}
$$

Therefore,

$$
\begin{aligned}
& \frac{d^{n}}{d t^{n}}\left[r(t) \int_{\Omega} Z_{i}(x, t) \phi(x) d x\right. \\
& \left.\quad+\int_{\alpha}^{\beta} p(t, \tau) \int_{\Omega} Z_{i}(x, t-\tau) \phi(x) d x d \tau\right] \\
& \leq a_{i}(t) \int_{\Omega} \Delta Z_{i}(x, t) \phi(x) d x \\
& \quad+\sum_{j=1}^{m} \sum_{k=1}^{s} b_{i j k}(t) \int_{\Omega} \frac{\delta_{j}}{\delta_{i}} \Delta Z_{j}\left(x, \rho_{k}(t)\right) \phi(x) d x
\end{aligned}
$$




$$
\begin{array}{r}
-\int_{\Omega} q_{i}(x, t) Z_{i}(x, t) \phi(x) d x \\
-\sum_{j=1}^{m} \sum_{\gamma=1}^{l} \frac{\delta_{j}}{\delta_{i}} \int_{\Omega} \int_{\mathcal{c}_{\gamma}}^{d_{\gamma}} h_{i j \gamma}(x, t) Z_{j}\left(x, t-\sigma_{\gamma}\right) d \sigma_{\gamma} \phi(x) d x, \\
t \geq t_{1}, \quad i \in I_{m} .
\end{array}
$$

Green's formula and boundary condition (4) yield

$$
\begin{aligned}
& \int_{\Omega} \Delta Z_{i}(x, t) \phi(x) d x=\int_{\Omega} Z_{i}(x, t) \Delta \phi(x) d x \\
& \quad=-\lambda_{0} \int_{\Omega} Z_{i}(x, t) \phi(x) d x \leq 0, \\
& \int_{\Omega} \Delta Z_{i}\left(x, \rho_{k}(t)\right) \phi(x) d x \\
& =\int_{\Omega} Z_{i}\left(x, \rho_{k}(t)\right) \Delta \phi(x) d x \\
& =-\lambda_{0} \int_{\Omega} Z_{i}\left(x, \rho_{k}(t)\right) \Delta \phi(x) d x \leq 0 .
\end{aligned}
$$

Combining (42)-(43), we get

$$
\begin{aligned}
& \frac{d^{n}}{d t^{n}}\left[r(t) \int_{\Omega} Z_{i}(x, t) \phi(x) d x\right. \\
& \left.\quad+\int_{\alpha}^{\beta} p(t, \tau) \int_{\Omega} Z_{i}(x, t-\tau) \phi(x) d x d \tau\right] \\
& \leq-\lambda_{0} \sum_{k=1}^{s} b_{i i k}(t) \int_{\Omega} \Delta Z_{i}\left(x, \rho_{k}(t)\right) \phi(x) d x \\
& \quad+\lambda_{0} \sum_{j=1, j \neq i}^{m} \sum_{k=1}^{s}\left|b_{i j k}(t)\right| \int_{\Omega} \Delta Z_{j}\left(x, \rho_{k}(t)\right) \phi(x) d x \\
& \quad-q_{i}(t) \int_{\Omega} Z_{i}(x, t) \phi(x) d x \\
& \quad-\sum_{\gamma=1}^{l} h_{i i \gamma}(t) \int_{\mathcal{C}_{\gamma}}^{d_{\gamma}} \int_{\Omega} Z_{i}\left(x, t-\sigma_{\gamma}\right) d \sigma_{\gamma} \phi(x) d x \\
& \quad+\sum_{j=1, j \neq i}^{m} \sum_{\gamma=1}^{l} \bar{h}_{i j \gamma}(t) \int_{c_{\gamma}}^{d_{\gamma}} \int_{\Omega} Z_{j}\left(x, t-\sigma_{\gamma}\right) \phi(x) d x d \sigma_{\gamma} \leq 0 .
\end{aligned}
$$

Set

$$
V_{i}=\int_{\Omega} Z_{i}(x, t) \phi(x) d x, \quad t \geq t_{1}, i \in I_{m} .
$$

Then (44) yields

$$
\begin{aligned}
& {\left[r(t) V_{i}(t)+\int_{\alpha}^{\beta} p(t, \tau) V_{i}(t-\tau) d \tau\right]^{(n)}} \\
& +\lambda_{0} \sum_{k=1}^{s}\left[b_{i i k}(t) \Delta V_{i}\left(x, \rho_{k}(t)\right)\right. \\
& \left.\quad-\sum_{j=1, j \neq i}^{m}\left|b_{i j k}(t)\right| \Delta V_{j}\left(x, \rho_{k}(t)\right)\right]+q_{i}(t) V_{i}(x, t) \\
& +\sum_{\gamma=1}^{l}\left[h_{i i \gamma \gamma}(t) \int_{\mathcal{c}_{\gamma}}^{d_{\gamma}} V_{i}\left(x, t-\sigma_{\gamma}\right) d \sigma_{\gamma}\right. \\
& \left.\quad-\sum_{j=1, j \neq i}^{m} \bar{h}_{i j \gamma}(t) \int_{\mathcal{c}_{\gamma}}^{d_{\gamma}} V_{j}\left(x, t-\sigma_{\gamma}\right) d \sigma_{\gamma}\right] \leq 0 .
\end{aligned}
$$

Let $V(t)=\sum_{i=1}^{m} V_{i}(t), t \geq t_{1}$; from (46) we have

$$
\begin{aligned}
& {\left[r(t) V(t)+\int_{\alpha}^{\beta} p(t, \tau) V(t-\tau) d \tau\right]^{(n)}} \\
& +\lambda_{0} \sum_{k=1}^{s}\left\{\sum _ { i = 1 } ^ { m } \left[b_{i i k}(t) \Delta V_{i}\left(x, \rho_{k}(t)\right)\right.\right. \\
& \left.\left.\quad-\sum_{j=1, j \neq i}^{m}\left|b_{i j k}(t)\right| \Delta V_{j}\left(x, \rho_{k}(t)\right)\right]\right\} \\
& +q_{i}(t) V(x, t) \quad \sum_{\gamma=1}^{l}\left\{\sum _ { i = 1 } ^ { m } \left[h_{i i i^{\prime}}(t) \int_{\mathcal{c}_{\gamma}}^{d_{\gamma}} V_{i}\left(x, t-\sigma_{\gamma}\right) d \sigma_{\gamma}\right.\right. \\
& \left.\left.-\sum_{j=1, j \neq i}^{m} \bar{h}_{i j \gamma}(t) \int_{\mathcal{c}_{\gamma}}^{d_{\gamma}} V_{j}\left(x, t-\sigma_{\gamma}\right) d \sigma_{\gamma}\right]\right\} \leq 0 .
\end{aligned}
$$

Now, as in the proof of Theorem 4, from (47) we have

$$
\begin{aligned}
{[r(t)} & \left.V(t)+\int_{\alpha}^{\beta} p(t, \tau) V(t-\tau) d \tau\right]^{(n)} \\
& +\lambda_{0} \sum_{k=1}^{s} B_{k}(t) V\left(\rho_{k}(t)\right)+q(t) V(t) \\
& +\sum_{\gamma=1}^{l} Q_{\gamma}(t) \int_{\mathcal{c}_{\gamma}}^{d_{\gamma}} V\left(t-\sigma_{\gamma}\right) d \sigma_{\gamma} \leq 0, \quad t \geq t_{1} .
\end{aligned}
$$

The remaining part of the proof is similar to that of Theorem 4; therefore it is omitted. The proof is complete. 
Corollary 10. If inequality (48) has no eventually positive solution, then every solution $u(x, t)$ of system (1), (4) is oscillatory in $G$.

It is not difficult to see that the following theorems are true.

Theorem 11. Assume that $r(t) \geq 1$ is a monotone decreasing function, and (9) holds. If there exists some $k_{0} \in I_{s}$ such that

$$
\int_{t_{0}}^{\infty}\left\{\lambda_{0} B_{k_{0}}(t)\left[1-\int_{\alpha}^{\beta} p\left(\rho_{0}(t), \tau\right) d \tau\right]\right\} d t=\infty, \quad t_{0}>0,
$$

then every solution of boundary value system (1), (4) is oscillatory in $G$.

Theorem 12. Suppose that there exists a positive constant $M$ such that $0<M \leq r(t)<1$, and (33) holds. If there exists some $k_{0} \in I_{s}$ such that

$$
\begin{array}{r}
\int_{t_{0}}^{\infty}\left\{\lambda_{0} B_{k_{0}}(t)\left[1-M^{-1} \int_{\alpha}^{\beta} p\left(\rho_{0}(t), \tau\right) d \tau\right]\right\} d t=\infty \\
t_{0}>0
\end{array}
$$

then every solution of boundary value system (1), (4) is oscillatory in $G$.

Theorem 13. Assume all conditions of Theorem 5 hold. Then every solution $u(x, t)$ of boundary value system (1), (4) is oscillatory in $G$.

Theorem 14. Assume all conditions of Theorem 7 hold. Then every solution $u(x, t)$ of boundary value system (1), (4) is oscillatory in $G$.

Theorem 15. Assume all conditions of Theorem 8 hold. Then every solution $u(x, t)$ of boundary value system (1), (4) is oscillatory in $G$.

\section{Example}

Example 1. Consider the systems of second-order partial differential equations

$$
\begin{aligned}
\frac{\partial^{2}}{\partial t^{2}}\left(\left(1+e^{-t}\right) u_{1}(x, t)+\frac{1}{2} \int_{\pi / 2}^{\pi} u_{1}(x, t-\tau) d \tau\right) \\
=\Delta u_{1}(x, t)+\left(\frac{3}{2}+3 e^{-t}\right) \Delta u_{1}\left(x, t-\frac{3 \pi}{2}\right)-\frac{3}{2} u_{1}(x, t) \\
\quad-\frac{e^{-t}+4}{2} \int_{\pi / 2}^{\pi} u_{1}\left(x, t-\sigma_{1}\right) d \sigma_{1} \\
\quad-\frac{e^{-t}}{2} \int_{\pi / 2}^{\pi} u_{2}\left(x, t-\sigma_{1}\right) d \sigma_{1}
\end{aligned}
$$

$$
\begin{gathered}
\frac{\partial^{2}}{\partial t^{2}}\left(\left(1+e^{-t}\right) u_{2}(x, t)+\frac{1}{2} \int_{\pi / 2}^{\pi} u_{2}(x, t-\tau) d \tau\right) \\
=e^{-t} \Delta u_{2}(x, t)+\left(\frac{3}{2}+2 e^{-t}\right) \Delta u_{2}\left(x, t-\frac{3 \pi}{2}\right)-\frac{5}{2} u_{2}(x, t) \\
-\frac{e^{-t}}{2} \int_{\pi / 2}^{\pi} u_{1}\left(x, t-\sigma_{1}\right) d \sigma_{1} \\
-\frac{e^{-t}+4}{2} \int_{\pi / 2}^{\pi} u_{2}\left(x, t-\sigma_{1}\right) d \sigma_{1} \\
(x, t) \in(0, \pi) \times[0, \infty)
\end{gathered}
$$

with boundary condition

$$
\frac{\partial}{\partial x} u_{i}(0, t)=\frac{\partial}{\partial x} u_{i}(\pi, t)=0, \quad t \geq 0, i=1,2 .
$$

Here, $N=1, n=2, m=2, \alpha=\pi / 2, \beta=\pi, k=1$, $c_{1}=\pi / 2, d_{1}=\pi, i=2, a_{1}(t)=1, a_{2}(t)=e^{-t}, q_{1}(x, t)=$ $3 / 2, q_{2}(x, t)=5 / 2, q_{111}(t)=\left(e^{-t}+4\right) / 2, q_{121}(t)=e^{-t} / 2$, $q_{212}(t)=e^{-t} / 2, q_{221}(t)=\left(e^{-t}+4\right) / 2, f_{i j \gamma}\left(x, t, u_{j}\right)=u_{j}$. It is easy to see that all conditions of Theorem 4 are fulfilled. Then every solution of system (52), (53) oscillates in $(0, \pi) \times[0, \infty)$. In fact, $u_{1}(x, t)=\cos x \sin t, u_{2}(x, t)=\cos x \cos t$. is such a solution.

\section{Conflict of Interests}

The authors declare that there is no conflict of interests regarding the publication of this paper.

\section{Acknowledgments}

The authors thank referees for both a careful reading of the paper and useful suggestions that helped to improve the presentation. This research is supported by Natural Sciences Foundation of China (no. 11172194) and Natural Sciences Foundation of Shanxi Province (no. 2010011008).

\section{References}

[1] W. N. Li, "Oscillation properties for systems and hyperbolic differential equations of neutral type," Journal of Mathematical Analysis and Applications, vol. 248, no. 2, pp. 369-384, 2000.

[2] W.-X. Lin, "A note on oscillation for systems of high order quasilinear partial differential equations of neutral type," Applied Mathematics and Computation, vol. 156, no. 2, pp. 563-576, 2004.

[3] W. N. Li, M. Han, and F. W. Meng, " $H$-oscillation of solutions of certain vector hyperbolic differential equations with deviating arguments," Applied Mathematics and Computation, vol. 158, no. 3, pp. 637-653, 2004.

[4] G. H. Gui and Z. T. Xu, "Oscillation of even order partial differential equations with distributed deviating arguments," Journal of Computational and Applied Mathematics, vol. 228, no. 1, pp. 20-29, 2009.

[5] L. H. Erbe, Q. Kong, and B. G. Zhang, Oscillation Theory for Functional-Differential Equations, vol. 190 of Monographs and 
Textbooks in Pure and Applied Mathematics, Marcel Dekker, New York, NY, USA, 1995.

[6] J. Wu, Theory and Applications of Partial Functional Differential Equations, Springer, New York, NY, USA, 1996.

[7] R. P. Agarwal, F. W. Meng, and W. N. Li, "Oscillation of solutions of systems of neutral type partial functional differential equations," Computers \& Mathematics with Applications, vol. 44, no. 5-6, pp. 777-786, 2002.

[8] W.-X. Lin, "Some oscillation theorems for systems of even order quasilinear partial differential equations," Applied Mathematics and Computation, vol. 152, no. 2, pp. 337-349, 2004.

[9] W. N. Li and B. T. Cui, "Necessary and sufficient conditions for oscillation of neutral delay parabolic differential equations," Applied Mathematics and Computation, vol. 121, no. 2-3, pp. 147153, 2001.

[10] W. N. Li and B. T. Cui, "Oscillation for systems of neutral delay hyperbolic differential equations," Indian Journal of Pure and Applied Mathematics, vol. 31, no. 8, pp. 933-948, 2000. 


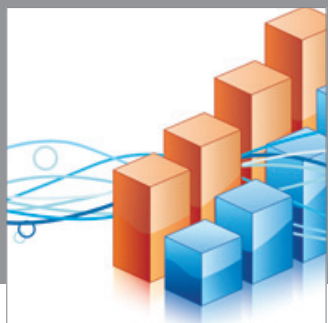

Advances in

Operations Research

mansans

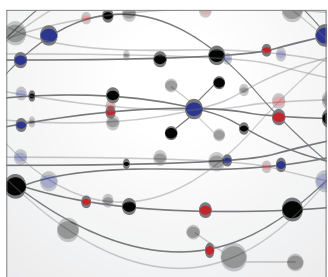

The Scientific World Journal
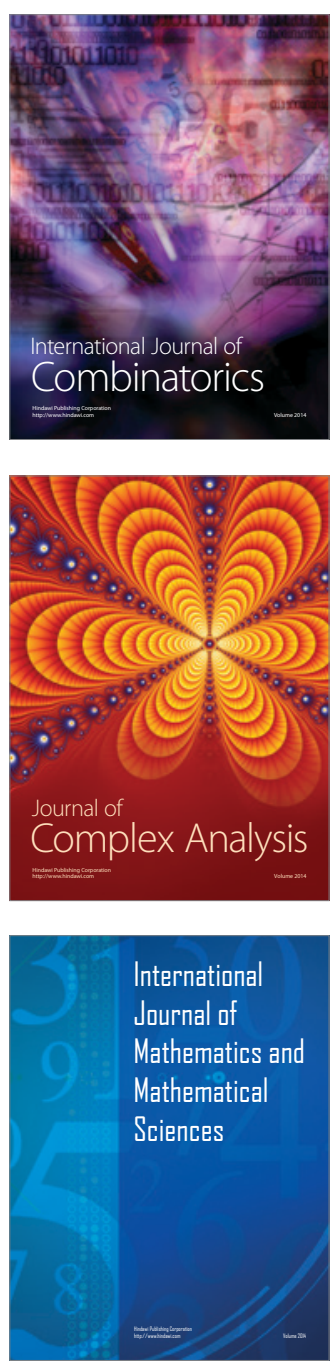
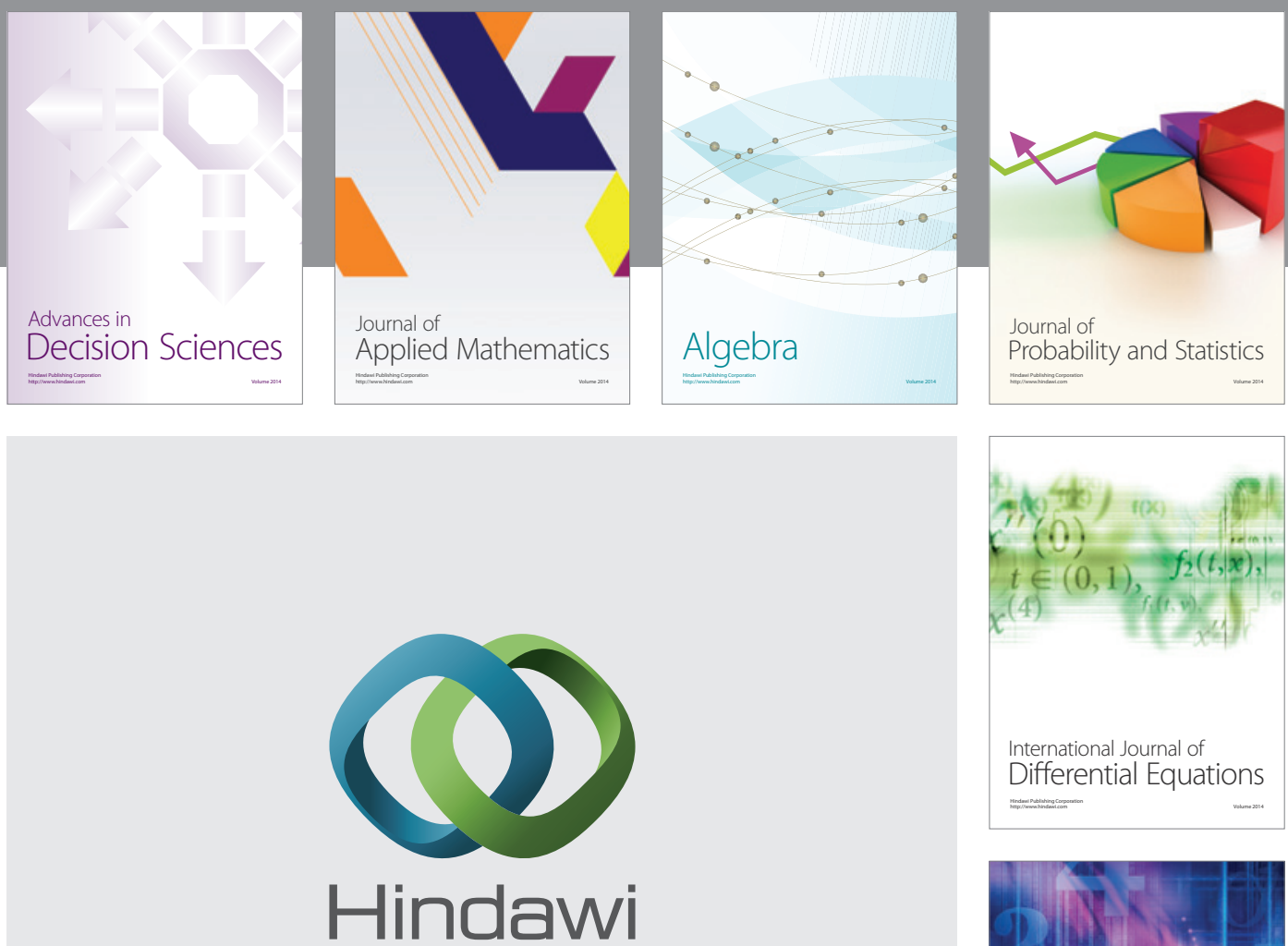

Submit your manuscripts at http://www.hindawi.com
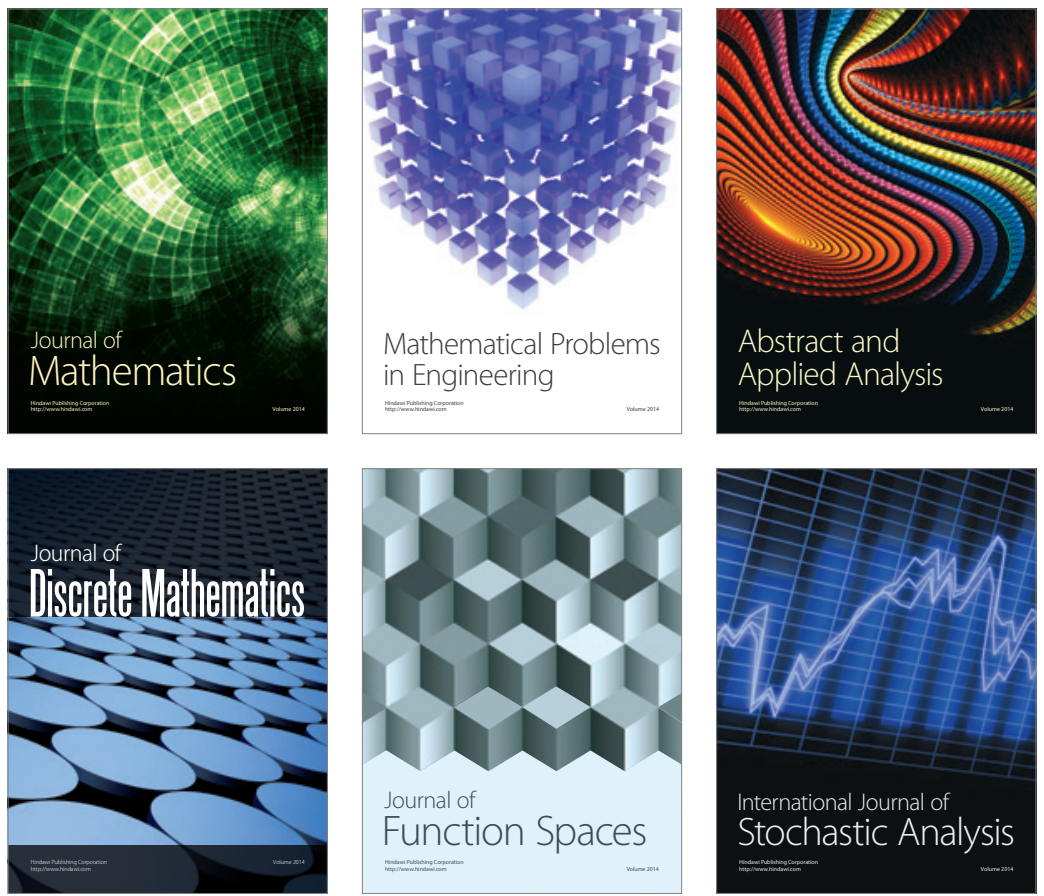

Journal of

Function Spaces

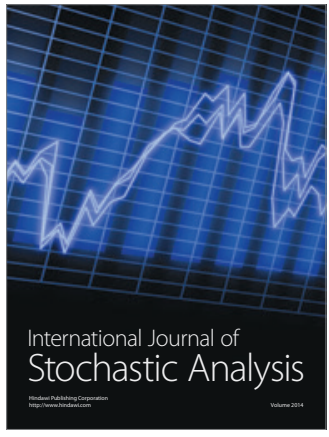

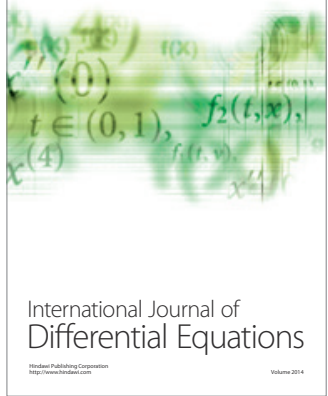
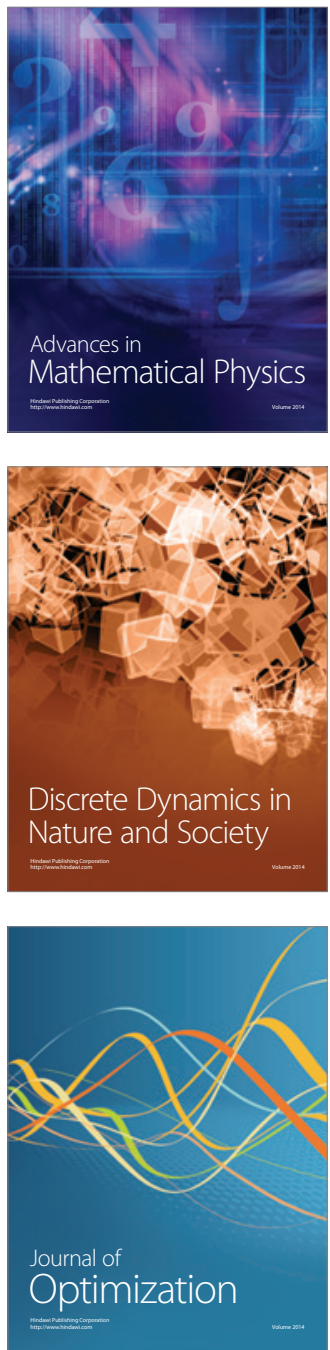